\title{
Metal allergy after the Nuss procedure for pectus excavatum: a review
}

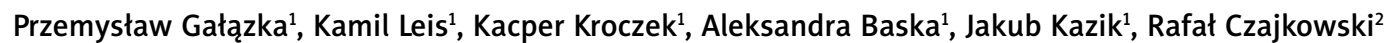

${ }^{1}$ Department of General and Oncological Surgery for Children and Adolescents, Antoni Jurasz University Hospital No. 1, Faculty of Medicine, Ludwik Rydygier Medical College in Bydgoszcz, Nicolaus Copernicus University in Torun, Poland ${ }^{2}$ Department of Dermatology and Venerology, Faculty of Medicine, Ludwik Rydygier Medical College in Bydgoszcz, Nicolaus Copernicus University in Torun, Poland

Adv Dermatol Allergol 2020; XXXVII (6): 848-852

DOI: https://doi.org/10.5114/ada.2020.102094

\begin{abstract}
Minimally invasive repair of pectus excavatum (MIRPE) technique (the Nuss procedure) is a minimally-invasive method that is commonly used in the treatment of pectus excavatum. An allergic reaction to the metal alloy bar that is implanted in the thorax during the procedure is a reported complication. We briefly review current literature concerning epidemiology, mechanisms and research results of allergic reactions after Nuss bar implantation. This allergic reaction occurs in approximately $2.7 \%$ of patients and is caused by metals used in the medical implant. The most common symptoms include fever and skin lesions such as allergic dermatitis. Elevated levels of C-reactive protein is a frequent finding in laboratory tests. In order to minimize the risk of such complications, taking a detailed allergy-based medical history and conducting allergy tests, i.e. patch test are required. Allergic reactions can be managed with conservative treatment such as general or topical glucocorticosteroid therapy and antihistamine agents. Severe allergic reactions can be addressed by implant revision, replacement of the steel bar with a titanium substitute or removal of the stabilization at all. Although the risk of an allergic reaction to titanium is smaller it still exists, the titanium substitute is not routinely used due to its higher cost and lesser plasticity which has a negative impact on matching a stabilizing bar during the surgery. Surgeons treating pectus excavatum should remember about the possible allergic reactions after implantation of the metal bar and be familiar with methods of diagnosis and treatment of those complications.
\end{abstract}

Key words: Nuss procedure, pectus excavatum, bar allergy, allergy testing.

\section{Introduction}

The Nuss procedure is a minimally-invasive method of pectus excavatum treatment. In 1987, Donald Nuss performed the first operation. It is based on the implantation of a concave stainless-steel bar with the use of a guideway into the thorax. Then, the bar is rotated 180 degrees, so the convexity is turned outward, thus restoring the natural physiological shape of the thorax. The bar is removed after 2-4 years. Despite the high efficiency and minimal invasiveness in comparison to the alternative methods (i.e. the Ravitch technique), the MIRPE technique carries the risk of side effects of an allergic reaction to metal [1-6].

\section{Mechanism}

An allergy to metals occurs in $10 \%$ of the general population. The symptoms usually occur as a result of direct contact. Metal ions bind with each other forming compounds with body's proteins, which leads to type IV hypersensitivity with Th CD4+ cells contribution. It is often incorrectly diagnosed as a post-operative infection [7, 8].

If the hypersensitivity-causing metal is nickel, the mechanism may be divided into several phases. During the first phase - the induction phase - the element is released from the metal (in this case, the bar) in a form of ions (haptens), which cross the skin and then bind to the dendritic cells. The dendritic cells present the ions to immunocompetent T cells causing their proliferation and subsequently inflammation (with the participation of Th CD8+ cells and produced cytokines). A maculopapular rash is usually the first skin symptom [7-11].

The nickel cations deposit as epitopes in the Langerhans cells, antigen-presenting cells (APCs) (metal binds to $M H C$ class II due to histidine $\beta$ chain) and dendritic

\footnotetext{
Address for correspondence: Przemysław Gałązka MD, PhD, Department of General and Oncological Surgery for Children and Adolescents, Antoni Jurasz University Hospital, Medical College, Nicolaus Copernicus University, 9 Sklodowskiej-Curie St, 85-094 Bydgoszcz, Poland, phone: +48 52585 4015, fax: +48 52585 4093, e-mail: galazkaprzemek@hotmail.com Received: 16.01.2020, accepted: 3.03.2020.
} 
cells. Subsequently, these complexes migrate to the peripheral lymph nodes. In response, CD4+ T cells, and to a lesser degree Th17 and Th22 cells, are produced and shifted to the skin. The activity of some lymphocytes may be induced without APC involvement due to direct antigen presentation. $T$ cell receptor $V$ beta repertoire of nickel specific $T$ cells and Toll like 4 receptors also take part in the hypersensitivity reaction. The latter stimulate the innate immune system [7-11].

Nowadays, there are a number of diagnostic tools that confirm allergy to metal. During the skin testing (the patch test), the skin reaction on a metal disc (offered by the Nuss bar's manufacturer) is examined. Another option is testing of metal in petrolatum. The previous method was found not to be adequately sensitive in preoperative screening (specifically looking for nickel allergy). It was found that patch tests using metal in petrolatum was a more sensitive and accurate means of preoperative nickel allergy testing [12]. Hypersensitivity to the skin test manifests most often within 48-96 h from exposure, but in some cases it may take up to $168 \mathrm{~h}$. The results of preoperative testing may be influenced by drugs, other allergens, active neoplastic disease, age, or inborn histamine intolerance $[12,13]$. Due to the risk of allergic reactions development in patients undergoing Nuss bar implantation and as a means of potentially avoiding reoperations, it has been suggested that patch tests should be used in all patients prior to the Nuss procedure $[12,14]$. On the other hand, in the review of Rosner and Fonacier based on the reasonable evidence and expert opinion, consensus guidelines for the evaluation of hypersensitivity to biomedical devices are presented. Namely authors mentioned that routine preimplantation evaluation in individuals with no history of adverse cutaneous reactions to metals or a history of implantrelated adverse events is not necessary. In case of unexplained symptoms, patients and clinicians may benefit from patch test evaluation although most of all, infection and biomechanical causes should be ruled out. The decision regarding implant revision can only be made after a thorough discussion among the patient, the allergist or dermatologist, and the surgeon [15].

The given allergen is dissolved in water or vaseline and then transferred to an aluminium chamber and applied to the upper or middle part of the back. After $48 \mathrm{~h}$ they are removed and the hypersensitivity results are estimated after the $2^{\text {nd }}, 3^{\text {rd }}, 6^{\text {th }}$, and $7^{\text {th }}$ day. The assessment is based on a four-grade scale (0 points - minimum, 3 points - maximum) [16]. Also other clinical scoring for signs of contact dermatitis are described in the literature [17].

For the detection of metal hypersensitivity in patients with implants lymphocyte transformation testing is also used. The test uses the measurement of lymphocytes in peripheral blood that are produced in the span of 7 days following allergen exposure [18]. The ratio of lymphocyte proliferation after allergen challenge to proliferation without the allergen is expressed as a stimulation index $[16,17]$. There are reported disadvantages to lymphocyte proliferation testing: high cost, limited availability, the limited number of allergens available for testing, lack of standardization, inter-laboratory variability, false-negative results in case of processing delay, and difficulty maintaining an appropriate sample for determination of lymphocyte proliferation $[16,17]$. This method however may provide some benefit for indeterminate or negative patch test results in a patient strongly suspected of having metal hypersensitivity. The use of the manufacturerprovided metal disc testing alone is limited by irritant, false negative and false positive reactions and is generally not recommended for clinical use $[16,17]$.

The risk factors of hypersensitivity development are: male sex (according to Rushing et al., around $82 \%$ of cases are boys), genetic predisposition and atopic dermatitis $[4,14,19]$. In some publications however an allergic reaction to a stainless steel bar or a positive patch test was more common in females [20]. It is also considered that polymorphisms in angiotensin converting enzyme (ACE), tumour necrosis factor (TNF), and N-acetyltransferase or filaggrin gene mutation may increase the chance of a pathological allergic reaction [16, 21-23].

The classical stainless-steel bar is composed of iron (over 60\%), chrome (17-19\%), nickel (13-15\%), molybdenum (2.5-3\%), manganese (2\%) and other elements like nitrogen, copper, or carbon. The primary cause of an allergic reaction inside the thorax is nickel or chrome and, to a lesser degree, other components.

Before beginning the Nuss procedure one should take a medical history and perform the skin (patch) test. In the case of a positive patch test result, the titanium bar should be implanted. If there are any allergic reactions during the post-operative period (the hypersensitivity symptoms develop approximately within 40 days, sometimes even later - within 140 days from the operation), glucocorticosteroid and histamine antagonists therapy should be introduced. When there is no improvement after the pharmacological treatment, the metal bar should be replaced with a titanium substitute. Many patients, in spite of confirmed hypersensitivity, do not manifest any side effects due to the metal bar presence $[4,6,13$, 16, 22].

The titanium bar consists of titanium (90\%), aluminium (5.5-6.75\%), vanadium (3.5-4.5\%), and other elements such as iron. Titanium is not used as the standard treatment method of pectus excavatum because it is far more expensive than nickel and chrome. It is also less flexible than other materials. The advantage of titanium bars is not only the allergic reaction reduction, but also it is not contraindicated during magnetic resonance imaging. It is important to point out that allergy to titanium also exists, but is much more uncommon [13, 16, 20-22]. Several scholars have reported cases of a suspected titanium allergy including cardiac pacemak- 
ers (with signs of pruritus, redness, and swelling of the skin overlying a titanium-containing pacemaker), failed total hip replacements and eczema upon titanium-based osteosynthesis [24]. Egusa et al. reported facial eczema in association with a titanium dental implant [25]. Siddiqi et al. wrote a review of the literature suggesting that titanium can induce hypersensitivity in susceptible patients and could have a critical role in implant failure [26]. In the study of Hosoki et al., the total number of allergypositive reactions for titanium allergens among all 270 patients was 17 (6.3\%). A total of 217 (80.4\%) patients exhibited allergy-positive reactions to at last one type of metal allergens. No patient exhibited a positive reaction only for the titanium allergen. Authors speculated that the prevalence of titanium allergy-positive cases in the normal population might be far less than stated in the presented study [27].

The clinical symptoms of metal sensitivity could be classified as local (maculopapular rash, oedema, erythema, keloid and erosions development) as well as systemic symptoms (fever, generalized exfoliative dermatitis, pericarditis) [4, 14-17]. Schalock et al. classified diagnostic criteria of metal hypersensitivity reactions as major (chronic dermatitis beginning weeks to months after metallic implantation, positive patch test reaction to metal used in the implant, eruption overlying the metal implant or complete recovery after removal of the offending implant) and minor (dermatitis reaction is resistant to therapy, morphology consistent with dermatitis, systemic allergic dermatitis reaction, histology consistent with allergic contact dermatitis, positive in vitro test result to metals - e.g., LTT). Interestingly, set numerical criteria required for diagnosis (such as the number of major and minor criteria to qualify) were not defined [16].

In 2016 Tao et al. ran an analysis on 828 patients who underwent the Nuss procedure. The control group had the classical bars with nickel and chrome implanted, while the experimental group received titanium/chitosan bars. The hypersensitivity to metal was assessed after 2 years of observation. In the control group there were 14 cases of allergy development, while in the experimental group there were none. The average of inflammation markers was lower in the group of patients with titanium bars in comparison to the group with classical bars [28].

\section{The research on allergy to metal}

In 2007, Rushing et al. published the results of an analysis involving 862 patients who underwent pectus excavatum surgical treatment, the Nuss procedure, between 1987 and 2005. Nineteen patients presented features of an allergic reaction. The average patients' age was 15, and 18 of them were male. Erythema and rash concurrent with granulomatous lesions or pleural effusion occurred in 10 patients. Atopy was diagnosed in 9 cases. A total of 3 patients required the replacement of the stabilizing bar due to necrotic skin lesions induced by metal. The symptoms of hypersensitivity disappeared as a result of reimplantation [4].

Kelly et al. in 2010 documented allergic reaction in 35 of 1215 patients who underwent the Nuss procedure treatment between 1987 and 2008. During the preoperative examination, hypersensitivity to nickel was diagnosed in 22 patients who as a result were implanted with a titanium substitute. Postoperatively 13 patients were diagnosed with allergic symptoms (10 patients were subjected to prednisolone therapy until the normalization of inflammatory markers, 2 patients had to have the original metal bar replaced with a titanium bar, 1 patient required the removal of a stabilizing bar without the necessity of reimplantation). The authors of the research claim that in almost $96 \%$ of cases, the surgery was effective and did not involve any complications [29].

In 2014, Shah et al. published an article showing that a total of 41 patients among 639 patients with pectus excavatum who were subjected to the minimally-invasive Nuss procedure were diagnosed with an allergy to metal. The research estimated the prevalence of allergic reactions at $6.4 \%$ which is a higher proportion compared with the results of older data [14].

Aneja et al. in 2011 published a report concerning 50 patients who were treated with the Nuss procedure. Postoperative adverse effects occurred in 4 cases: 3 patients presented with postoperative wound granulation and 1 patient presented with oedema and peripheral lymphadenopathy. Two patients were subjected to allergic tests and, as a result, hypersensitivity to nickel was confirmed in 1 patient [30].

A similar analysis was conducted in 2018 by Obermeyer et al. The research involved 932 patients, divided into 2 groups, who underwent the Nuss procedure. The first group included the cases from 2004-2011 ( $n=628)$ where metal allergy tests were performed only in special cases $(n=63)$. The second group consisted of the patients from 2011-2014 $(n=304)$ and all of them were subjected to allergy tests. In a total of 842 patients, steel bars were used during the procedure and 15 of them required further replacement of the original stabilizing bar due to allergic reaction. Interestingly, the hypersensitivity prevalence was similar in both groups and totalled respectively: $1.8 \%$ and $1.7 \%$. According to the researchers, an allergy to metal statistically develops more often in people with known family hypersensitivity and more frequently occurs in women [22].

Shu et al. in 2011 presented a comparison of 406 patients who were subjected to the Nuss procedure. A total of 313 patients were male, 93 patients were female and the average age was 7 . Only 2 cases presented with an allergic reaction. The alleviation of hypersensitivity symptoms was achieved in 1 patient by the removal of the stabilizing bar while the second patient presented with good effects of pharmacological treatment [31]. 
The literature offers many more analyses concerning an allergy to metal occurrence in patients subjected to the Nuss procedure. Although not so extensive, however, these studies indicate the prevalence rate of this complication. In 2016, Nuss et al. published the results of 1463 surgeries performed between 1987 and 2012. Hypersensitivity reactions to the implanted stabilizing bars occurred in 39 cases which equals to $2.7 \%$ [32]. Wang et al. collected 221 cases of patients who were surgically treated due to pectus excavatum ( 203 by the Nuss procedure and 18 by the Ravitch procedure) from 2005 to 2009 . The majority of patients $(n=189)$ were male and the average age was 8 . The allergic symptoms occurred in 2 patients [33].

Currently, there is still a debate concerning the need of pre-implant testing in orthopaedic surgery as testing has not consistently been shown to change patient outcomes [18].

\section{The summary of the results of the research}

The results of the previously described analyses are presented in Table 1. It includes 5680 patients with a congenital thoracic wall deformity - pectus excavatum who were subjected to the Nuss procedure. A total of 154 patients presented with the symptoms of allergy to metal as a result of classical implantation of a stabilizing nickel or chrome bar, which equals to $2.7 \%$. The research conducted by Obermeyer et al. in 2018 is the only study which estimated the prevalence rate of hypersensitivity reactions at more than $3 \%$, which was acknowledged by the authors [22]. The results show that there was not a single case of patient death or any severe, permanent health complications. The literature indicates that the most severe effects of an allergy to a metal are: pleuritis and pericarditis. These symptoms occur rarely. The most common manifestations of an allergy were rash, fever and an elevation of inflammation markers. The results indicate that the Nuss procedure is a safe surgical technique and a rapid reaction involving implementation of a pharmacological treatment based on steroids or the removal of a stabilizing metal bar (and its replacement with a titanium substitute in majority of cases) resulted in remission of all hypersensitivity symptoms.

\section{Conclusions}

An allergy to metal is relatively frequent and estimated at $10 \%$ of the general population. After the Nuss procedure, the allergy occurs in $0.5-6.4 \%$ of patients. For comparison, in dermatology it occurs in over $18 \%$ of cases and in orthopaedics in about $13 \%$ of cases [14]. It usually concerns nickel and chrome which are the metals used in a standard stabilizing bar. In order to avoid this complication, allergy patch tests are performed. In case of the occurrence of postoperative symptoms, a pharmacological glucocorticoid therapy and the stabilizing bar
Table 1. The summary of the research on the allergy to metal as a result of a classical implantation of a stabilizing nickel or chrome bar in the pectus excavatum patients

\begin{tabular}{lccc}
\hline Research & Patients & $\begin{array}{c}\text { Cases of } \\
\text { allergy }\end{array}$ & Percentage \\
\hline Rushing et al. 2007 & 862 & 19 & 2.2 \\
\hline Kelly et al. 2010 & 1215 & 35 & 2.8 \\
\hline Shah et al. 2014 & 639 & 41 & 6.4 \\
\hline Aneja et al. 2011 & 50 & 1 & 2 \\
\hline Obermeyer et al. 2018 & 842 & 15 & 1.8 \\
\hline Shu et al. 2011 & 406 & 2 & 0.5 \\
\hline Nuss et al. 2016 & 1463 & 39 & 2.7 \\
\hline Wang et al. 2009 & 203 & 2 & 1 \\
\hline Total & 5680 & 154 & 2.7 \\
\hline
\end{tabular}

replacement with a titanium substitute provide good results. There is ongoing research on other methods that could be useful in the prevention of allergic reactions. Overlaying the standard metal bar with a layer of titanium and chitosan seems highly effective but for the time being there are only individual cases that confirm the efficacy of this method. Despite the risk of intraoperative and postoperative complications thoracoscopic correction of pectus excavatum by the Nuss procedure is the method of choice.

\section{Conflict of interest}

The authors declare no conflict of interest.

\section{References}

1. Nuss D. Recent experiences with minimally invasive pectus excavatum repair "Nuss procedure”. Jpn J Thorac Cardiovasc Surg 2005; 53: 338-44.

2. NusS D, Kelly RE. Indications and technique of Nuss procedure for pectus excavatum. Thorac Surg Clin 2010; 20: 583-97.

3. Krasopoulos G, Dusmet M, Ladas G, et al. Nuss procedure improves the quality of life in young male adults with pectus excavatum deformity. Eur J Cardiothorac Surg 2006; 29: 1-5.

4. Rushing GD, Goretsky MJ, Gustin T, et al. When it is not an infection: metal allergy after the Nuss procedure for repair of pectus excavatum. J Pediatr Surg 2007; 42: 93-7.

5. Sakamoto K, Ando K, Noma D. Metal allergy to titanium bars after the Nuss procedure for pectus excavatum. Ann Thorac Surg 2014; 98: 708-10.

6. Navratil M, Batinica M, Ivković-Jureković I. Metal allergy as a late-onset complication of the Nuss procedure in a pediatric patient. Pediatr Pulmonol 2018; 53: E24-6.

7. Silverberg NB, Licht J, Friedler S, et al. Nickel contact hypersensitivity in children. Pediatr Dermatol 2002; 19: 110-3.

8. Dhingra N, Shemer A, da Rosa JC, et al. Molecular profiling of contact dermatitis skin identifies allergen-dependent differences in immune response. J Allergy Clin Immunol 2014; 134: 362-72. 
9. Girolomoni G, Gisondi P, Ottaviani C, et al. Immunoregulation of allergic contact dermatitis. J Dermatol 2004; 31: 264-70.

10. Peana M, Zdyb K, Medici S, et al. Ni (II) interaction with a peptide model of the human TLR4 ectodomain. J Trace Elem Med Biol 2017; 44: 151-60.

11. Lu L, Vollmer J, Moulon C, et al. Components of the ligand for a $\mathrm{Ni++}$ reactive human T cell clone. J Exp Med 2003; 197: 567-74.

12. Heitmiller K, French A, Alaish SM, et al. Patch testing for metal allergy with manufacturer-supplied materials before Nuss bar insertion. Dermatitis 2015; 26: 271-5.

13. Sesia SB, Haecker FM, Shah B, et al. Development of metal allergy after Nuss procedure for repair of pectus excavatum despite preoperative negative skin test. J Pediatr Surg Case Rep 2013; 1: 152-5.

14. Shah B, Cohee A, Deyerle A, et al. High rates of metal allergy amongst Nuss procedure patients dictate broader preoperative testing. J Ped Surg 2014; 49: 451-4.

15. Rosner GA, Fonacier LS. Hypersensitivity to biomedical implants: prevention and diagnosis. Allergy Asthma Proc 2017; 38: 177-83.

16. Schalock PC, Crawford G, Nedorost S, et al. Patch testing for evaluation of hypersensitivity to implanted metal devices: a perspective from the American Contact Dermatitis Society. Dermatitis 2016; 27: 241-7.

17. Schalock PC, Menne T, Johansen JD, et al. Hypersensitivity reactions to metallic implants - diagnostic algorithm and suggested patch test series for clinical use. Contact Dermatitis 2011; 66: 4-19.

18. Richards LJ, Streifel A, Rodrigues JM. Utility of patch testing and lymphocyte transformation testing in the evaluation of metal allergy in patients with orthopedic implants. Cureus 2019; 11: e5761.

19. Selvick A, Lloyd R. Patch Testing for the evaluation of metal hypersensitivity in the Nuss procedure. Dermatitis 2018; 29: 63-5.

20. Aneja S, Taylor JS, Sood A, et al. Concepts in allergy to pectus metal implants. In: Chest Wall Deformities. Saxena AK (ed.). Springer, Berlin 2017; 307-11.

21. Almutairi N, Almutawa F. The role of nickel allergy in hand dermatitis and its impact on handling cupronickel currency coins. A comparative cohort study from Kuwait. Adv Dermatol Allergol 2017; 34: 313-21.

22. Obermeyer RJ, Gaffar S, Kelly Jr RE, et al. Selective versus routine patch metal allergy testing to select bar material for the Nuss procedure in 932 patients over 10 years. J Ped Surg 2018; 53: 260-4.

23. Reduta T, Bacharewicz J, Pawlos A. Patch test results in patients with allergic contact dermatitis in the Podlasie region. Adv Dermatol Allergol 2013; 30: 350-7.

24. Fage SW, Muris J, Jakobsen SS, et al. Titanium: a review on exposure, release, penetration, allergy, epidemiology, and clinical reactivity. Contact Dermatitis 2016; 74: 323-45.

25. Egusa H, Ko N, Shimazu T, et al. Suspected association of an allergic reaction with titanium dental implants: a clinical report. J Prosthet Dent 2008; 100: 344-7.

26. Siddiqi A, Payne AG, De Silva RK, et al. Titanium allergy: could it affect dental implant integration? Clin Oral Implants Res 2011; 22: 673-80.

27. Hosoki M, Nishigawa K, Tajima T, et al. Cross-sectional observational study exploring clinical risk of titanium allergy caused by dental implants. J Prosthodont Res 2018; 62: 426-31.
28. Tao Y, Huang Y, Li J, et al. Titania/chitosan coated implants as a new solution for metal allergy and postoperative infection in pectus excavatum patients after Nuss surgery. J Biomater Tissue Eng 2016; 6: 975-82.

29. Kelly RE, Goretsky MJ, Obermeyer R, et al. Twenty-one years of experience with minimally invasive repair of pectus excavatum by the Nuss procedure in 1215 patients. Ann Surg 2010; 252: 1072-81.

30. Aneja S, Taylor JS, Soldes O, et al. Dermatitis in patients undergoing the Nuss procedure for correction of pectus excavatum. Contact Dermatitis 2011; 65: 317-21.

31. Shu Q, Shi Z, Xu WZ, et al. Experience in minimally invasive Nuss operation for 406 children with pectus excavatum. World J Pediatr 2011; 7: 257-61.

32. Nuss D, Obermeyer RJ, Kelly RE. Nuss bar procedure: past, present and future. Ann Cardiothorac Surg 2016; 5: 422-33.

33. Wang X, Xu B, Liu W, et al. Complication and treatment for correction of pectus excavatum with Nuss procedure. Zhongguo Xiu Fu Chong Jian Wai Ke Za Zhi 2009; 23: 1343-6. 